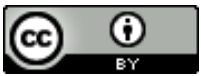

\title{
Zoo Foraging Ecology: Preference and Welfare Assessment of Two Okapi (Okapia johnstoni) at the Brookfield Zoo
}

\author{
Sandra M. Troxell-Smith' ${ }^{1 *}$, Jason V. Watters ${ }^{2}$, Christopher J. Whelan', \\ and Joel S. Brown ${ }^{1,3}$ \\ ${ }^{1}$ University of Illinois at Chicago, Chicago, IL, USA \\ ${ }^{2}$ San Francisco Zoological Society, San Francisco, CA, USA \\ ${ }^{3}$ Moffitt Cancer Center, Tampa, FL, USA \\ * Corresponding author (Email: sandra.troxell@gmail.com)
}

Citation - Troxell-Smith, S. M., Watters, J. V., Whelan, C. J., \& Brown, J. S. (2017). Zoo foraging ecology: Preference and welfare assessment of two okapi (Okapia johnstoni) at the Brookfield Zoo. Animal Behavior and Cognition, 4(2), 187-199. https://doi.org/10.12966/abc.05.05.2017

\begin{abstract}
Applying principles of foraging ecology to zoo-housed animals can positively influence animal behavior, and assist with evaluating exhibit space and design. In this study, we implemented zoo foraging ecology by measuring giving-up densities (GUDs) in food patches to address several welfare-related questions with captive okapi (Okapia johnstoni). Our objectives were to: (1) determine whether food patches can reveal how the individual animals perceive their exhibit space (i.e., areas of preference and aversion; landscapes of comfort); and (2) determine whether implementation of food patches could reduce performance of repetitive behavior. We established 24 food patches throughout the $929 \mathrm{~m}^{2}$ outdoor exhibit, determined each okapi's landscape of comfort, and evaluated the effects of the presence or absence of these food patches on okapi behavior. Food patches revealed landscapes of comfort that were unique to each individual. Food patch presence did not significantly lower the proportion of time spent in repetitive behavior, but did significantly increase the proportion of time that each animal spent actively foraging and animal movement throughout the exhibit space. We conclude that utilizing food patches in animal enrichment and welfare regimes can benefit zoo-housed species, particularly okapi, by providing not only a valuable form of enrichment, but by also allowing animals to directly reveal their individual perceptions and exhibit preferences to their caretakers.
\end{abstract}

Keywords - Animal welfare, Behavioral enrichment, Giving-up density, Okapi, Repetitive behavior

In the wild, animals must assess and respond to their heterogeneous and constantly changing environment. Resources in nature vary spatially and temporally, requiring animals to assess and adjust habitat use, foraging tactics, and time allocation appropriately (Charnov, 1976). For animals in captive situations, however, the environment is far less complex, and often quite predictable. Regulated cleaning, feeding, and management routines, coupled with reduced environmental complexity, may limit animals' opportunities to perform "natural" behaviors (Carlstead, 1996; McPhee, 2003; Shepherdson, 2010). Chronic suppression of behavioral expression may result in suboptimal welfare (Morgan \& Tromborg, 2007), including the expression of abnormal or repetitive behaviors (Mason \& Latham, 2004; Mason \& Veasey, 2010; Swaisgood \& Shepherdson, 2005). Repetitive behaviors (i.e., behaviors that are invariant, 
highly repetitive, and do not serve an obvious function; Mason, 1991), are thought to occur when captive animals are exposed to an ecologically relevant problem that they are incapable of solving within their enclosure (Shyne, 2006).

In a simultaneous effort to increase both the environmental complexity of captive exhibits, and the natural behavioral diversity of the animals within them, zoos routinely provide various forms of environmental enrichment (Chamove, 1989; Shepherdson, 2010). In particular, many wild species spend a significant proportion of their daily time budget on foraging-related activities (Herbers, 1981), which is directly at odds with the highly predictable manner in which food is provided to animals in captivity (Kistler, Hegglin, Würbel, \& König, 2009; McPhee \& Carlstead, 2010; Newberry, 1995). Consequently, many captive facilities implement enrichment programs designed to extend the amount of time animals spend on foraging-related activities. For example, food items may be dispersed throughout an exhibit rather than isolated to one area (Hare et al., 2003; Ryan, Proudfoot, \& Fraser, 2012). Food may also be hidden in various locations (Carlstead, Seidensticker, \& Baldwin, 1991; Ings, Waran, \& Young, 1997), or distributed according to varied feeding schedules (Kistler et al., 2009; Schneider, Nogge, \& Kolter, 2014; Watters, Miller, \& Sullivan, 2011) to increase the amount of time it takes animals to find and acquire their food. Similarly, providing carnivores with whole carcasses rather than processed meat can increase the handling time associated with food consumption (Bond \& Lindburg, 1990; McPhee, 2002; Ruskell, Meiers, Jenkins, \& Santymire, 2015).

To date, foraging enrichment is typically implemented within captive facilities with two main goals - to promote natural foraging behavior and increase foraging times. However, by integrating carefully designed feeding methodologies with concepts from foraging ecology and theory, caretakers and researchers can not only increase the foraging times of their animals, but can also gain insight into the perceptions and well-being of animals in their care. Recent "zoo foraging ecology" studies have begun to address a variety of animal welfare-related questions by utilizing ideas rooted in foraging ecology. For example, by placing several identical experimental food patches throughout a zoo exhibit, studies have revealed the environmental preferences and aversions of several zoo-housed species, including armadillos (Tolypeutes matacus; Howell-Stephens, 2012), zebra (Equus burchelli), bison (Bison bison bison), rock hyrax (Procavia capensis; Mogerman, 2011), Patagonian maras (Dolichotis patagonum), and Parma wallabies (Marcopus parma; Troxell-Smith, Whelan, Magle, \& Brown, in press). Quantification of these areas were then used to document and reflect an animal's "landscape of comfort" (i.e., an environmental "map" of areas of preference and aversion; Mogerman, 2011; Troxell-Smith et al., in press) within its exhibit. Food patches have also been found to provide a valuable enrichment activity - zoo-housed Patagonian maras and Parma wallabies increased the time spent foraging, decreased inactive behaviors, and increased utilization of exhibit space when food patches were provided when compared with traditional foraging methods (Troxell-Smith et al., in press). Such results highlight the potential for food patches to positively impact care and welfare decisions for animals in captivity.

Here, our objectives were to expand upon the existing zoo foraging ecology framework to explore two new applications of zoo foraging ecology: (1) evaluate to what extent individual animals within a species differ in their environmental preferences within the same exhibit space, and (2) to determine whether the use of food patches can reduce performance of repetitive behavior in zoo animals. This study was performed with two okapi at the Brookfield Zoo in Brookfield, IL, USA. As individual animals likely vary in their perception and interpretation of environmental stimuli (Réale, Reader, Sol, McDougall, \& Dingemanse, 2007), we predicted that each okapi would demonstrate distinct landscapes of comfort (i.e., each individual would prefer and avoid different areas) within the same exhibit. Regarding behavior, we predicted that the okapi would perform fewer repetitive behaviors, and would increase time spent foraging and movement throughout the exhibit when provided with food patches compared to traditional feeding methods. 


\section{Method}

This study was approved by the UIC Office of Animal Care and Institutional Biosafety (OACIB; protocol \# 13-152), and the Brookfield Zoo Biological Research Steering Committee (BRSC; protocol \# 322).

\section{Subjects and Housing}

The okapis were chosen for this study due to their noted performance of repetitive behaviors, and because both individuals were housed in the same exhibit space but at different times. Our two subjects were an adult female okapi (born at the Brookfield Zoo in April 2011), and an adult male okapi (born at the San Diego Zoo in March 1996, but who had resided at the Brookfield Zoo since June 2011). As okapi are primarily solitary in the wild (Bodmer \& Rabb, 1992; Hart \& Hart, 1988), the Brookfield Zoo housed the animals individually. We therefore collected data on the female between June-September 2013, and the male between June-July 2014, when each individual had access to the "Entry Yard", the largest available outdoor exhibit space for the okapis $\left(929 \mathrm{~m}^{2}\right)$. The okapi were on exhibit daily between approximately 0900-1700h. When not on exhibit, animals were housed in individual stalls in an indoor holding barn. Each indoor stall allowed visual access (but no physical access) to other okapi, and contained various rotating enrichment items.

In both feeding conditions, the okapi were primarily fed Mazuri Wild Herbivore diet ${ }^{\circ}$ (PMI Nutrition International). On normal feeding days (traditional methods), each animal's daily ration (2400 g) was divided into three meals: morning, mid-day, and evening. Each meal $(800 \mathrm{~g})$ was provided for the animals in a single feeding trough attached to the exhibit fence, and was typically completely consumed within a few minutes. The location of the trough was altered each day. Within this same trough, okapi were also provided with a ration of chopped vegetables and/or fruits during morning and evening feedings. In addition, okapi had ad libitum access to alfalfa hay throughout the day. During days with food patches, the entire pelletized diet ration was provided only in the food patches (as described below) in the morning. Hay was still provided, but fruits and vegetables were held until after the animals were brought into the holding barn for the night.

\section{Food Patches}

To ensure familiarity with the food patches, each animal had approximately three weeks to acclimate to the food patches before data collection began. Each food patch consisted of a 19L plastic bucket divided into two levels by a plastic plate. Each level was accessible to the okapi via an irregular hole ( $\sim 15 \mathrm{~cm}$ diameter; one on each level), and contained $50 \mathrm{~g}$ of grain pellets (100g total $/ \mathrm{patch})$ and five, softball-sized wiffle ${ }^{\circledR}$ balls (Wiffle Ball, Inc., Shelton, CT, USA; Figure 1A). The wiffle balls serve as an inedible substrate that increases foraging difficulty and ensures diminishing returns (i.e., as the animal forages, each piece of food becomes progressively harder to find; Brown, 1988). Food patches were placed in the exhibit in the morning $(0900 \mathrm{hr})$, and were collected in the evening (1700 hr), allowing an approximately $8 \mathrm{hr}$ foraging period. Food remaining in each bucket at the end of the day was separated from the whiffle balls and weighed, providing the giving-up density (GUD; Brown, 1988). Any leftover food was then provided to each okapi in the evening to ensure that they had the opportunity to consume their entire daily ration.

\section{Landscape of Comfort}

Under natural conditions, okapi move along well established paths through a diverse landscape, as individuals travel among shrubs or trees in search of a variety of browse (Hart \& Hart, 1988). To mimic the okapis' natural, above-ground feeding conditions, food patches were attached approximately 2 
$\mathrm{m}$ off the ground to tree trunks using cable ties (Figure 1B). On days with food patches, one food patch was secured to each of the 24 trees of the exhibit space. 10 days of food patch data were collected from the female okapi in 2013 for a total of 240 GUD measures. Seven days of food patch data were collected from the male okapi in 2014 for a total of 168 GUD measures.
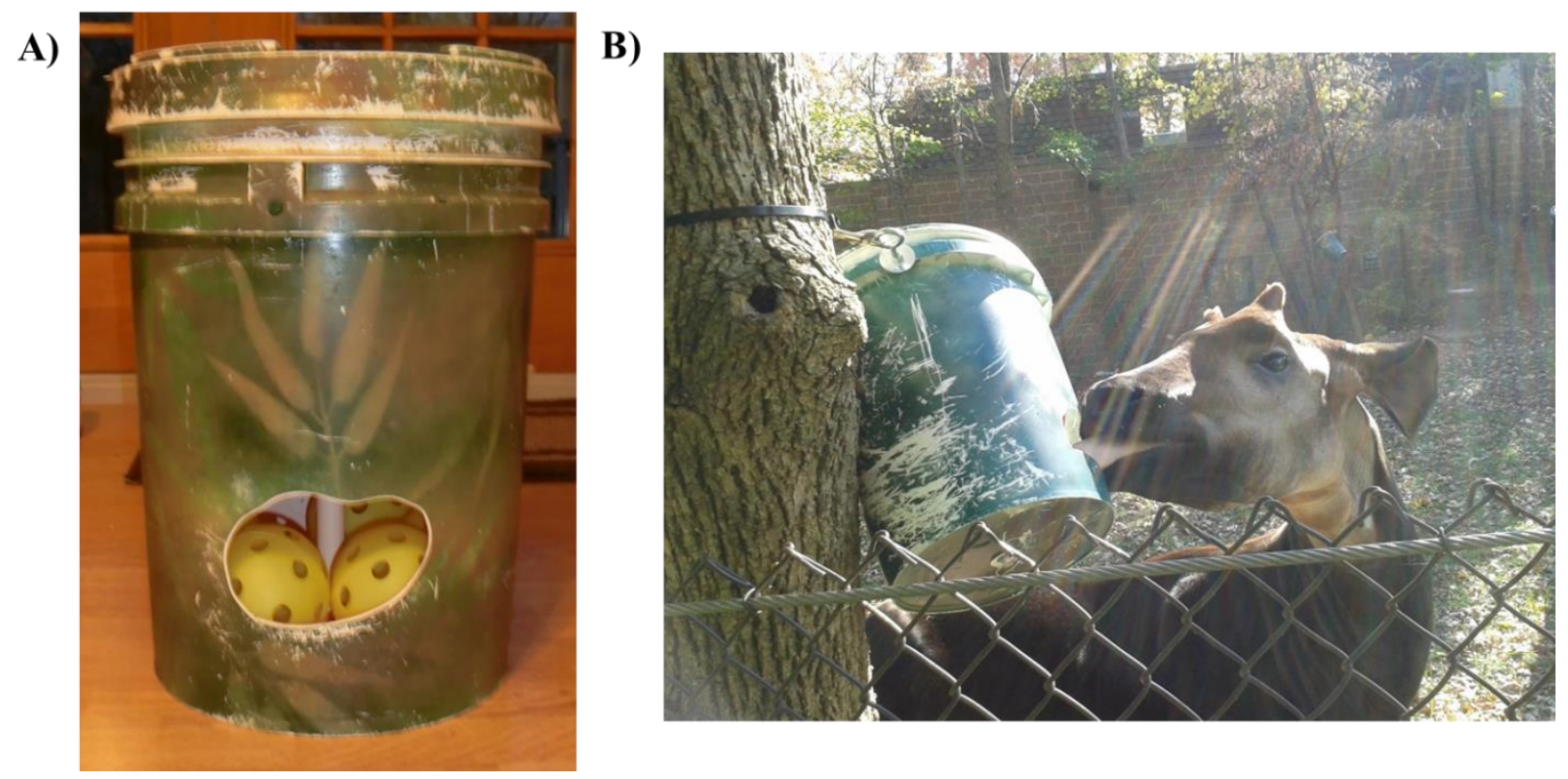

Figure 1. Photographs of okapi food patches. A) Close up of a food patch highlighting the bottom level containing five wiffle balls. B) The male okapi using his prehensile tongue to feed from a food patch in the exhibit. Food patches were attached to the tree approximately $2 \mathrm{~m}$ off the ground via cable ties to simulate folivorous feeding.

\section{Behavioral Observations}

Prior to data collection, we conducted behavioral observations on the okapi to generate a speciesspecific ethogram. A total of 23 behaviors were defined (see Table 1), including repetitive behaviors unique to each individual. The female's predominate repetitive behaviors were "head-rolling" and "pacing", whereas the male primarily performed a "mouthing" behavior (for behavioral definitions, see Table 1). When official data collection began, at least two $30 \mathrm{~min}$ observations were conducted each observation day during two time periods; one in the morning $(0900-1230 \mathrm{hr})$ and one in the afternoon (1231 - $1700 \mathrm{hr}$ ). Instantaneous sampling (Altmann, 1974) of state behaviors and animal location within the exhibit were recorded at $1 \mathrm{~min}$ fixed intervals. We used a random number generator in Microsoft Excel to assign specific treatment days and observation times within morning and afternoon periods. Data were recorded using the Animal Behavior Pro app for iPad (Newton-Fisher, 2012, University of Kent).

Between June-September 2013, total observation time for the female okapi was 25 days; 10 days (49.5 hrs) with food patches and 15 days $(24.6 \mathrm{hrs})$ without food patches. Due to weather and limitations imposed by various animal care and management decisions, the male okapi received 14 days (totaling 39 hrs) of observation between June - July 2014; 7 days (25.2 hrs) with food patches, and 7 days (13.7 hrs) without food patches. 
Table 1

Okapi Ethogram of Observed Behaviors and Corresponding Behavioral Categories

\begin{tabular}{|c|c|c|}
\hline $\begin{array}{l}\text { Behavioral } \\
\text { Category }\end{array}$ & Behavior & Definition \\
\hline \multirow[t]{4}{*}{ Active } & Attentive & Sudden turning of head and ears toward a direction. No other movement. \\
\hline & Running & Fast locomotion throughout exhibit. \\
\hline & Sniffing & Nose pointed toward object accompanied by rapid head movement. \\
\hline & Walking & Slow locomotion throughout exhibit. \\
\hline \multirow[t]{7}{*}{ Foraging } & Browse (Enrichment) & Consumption of browse provided by keepers for enrichment. \\
\hline & Browse (Fence) & $\begin{array}{l}\text { Consumption of native plants/browse found in and near exhibit borders. Not } \\
\text { provided by keepers. }\end{array}$ \\
\hline & Chewing & Grinding of food (browse, grain, or hay) with teeth. \\
\hline & Drinking & Consumption of water. \\
\hline & Enrichment (Hay Ball) & $\begin{array}{l}\text { Investigation and interaction with enrichment item holding hay. Present } \\
\text { during both traditional and food patch feeding. }\end{array}$ \\
\hline & Food Patch & $\begin{array}{l}\text { Investigation and interaction with a food patch, including removal and } \\
\text { consumption of pellets. Only used when food patches were available. }\end{array}$ \\
\hline & Traditional Feeder & $\begin{array}{l}\text { Investigation and interaction with traditional food trough, including } \\
\text { consumption of food pellets. Only used when food was presented in } \\
\text { traditional manner. }\end{array}$ \\
\hline \multirow[t]{6}{*}{ Maintenance } & Defecation/Urination & Waste excretion. \\
\hline & Headshake & Quick head movement from side to side (usually to remove flies). \\
\hline & Lying down & $\begin{array}{l}\text { Animal has all parts of body (apart from head) in contact with the ground. } \\
\text { No other movement occurs. }\end{array}$ \\
\hline & Rubbing & Uses branch to scratch head/ears. \\
\hline & Ruminate & Sudden chewing without recent prior consumption of food. \\
\hline & Self-groom & Using tongue to lick body (ears, back, legs, etc.). \\
\hline \multirow[t]{6}{*}{ Repetitive } & Head Rolling & $\begin{array}{l}\text { Moves head in circular pattern while facing vertical surface. Commonly } \\
\text { accompanied by alternate stomping on front right and hind left leg. Only } \\
\text { displayed by female okapi. }\end{array}$ \\
\hline & Inactive & $\begin{array}{l}\text { Upright on all four legs, but no other movement occurs. Ears are not fixed in } \\
\text { a particular direction. }\end{array}$ \\
\hline & Licking & $\begin{array}{l}\text { Using tongue to touch objects in exhibit. Does not include self-grooming. } \\
\text { Performed by both okapi. }\end{array}$ \\
\hline & Mouthing & $\begin{array}{l}\text { Rubs mouth on object (fence, enrichment, etc). Does not include self- } \\
\text { grooming. Only displayed by male okapi. }\end{array}$ \\
\hline & Pace & Repetitive walking pattern (not toward a destination). \\
\hline & Not Visible & Animal is not visible to observer. \\
\hline
\end{tabular}




\section{Data Analysis}

Time budget/behavioral enrichment. For analysis, we specifically focused on the two pertinent behavioral categories of time spent Foraging and time spent on Repetitive behaviors. Time spent in each of these behavioral categories in the morning and in the afternoon was calculated for each animal as proportion of time that the animal was visible to the observer. To test for the effect of presence and absence of food patches on animal movement throughout the exhibit, we divided the exhibit into sections based on fixed visual cues found within the exhibit (e.g., trees, fence sections, etc.). We then calculated the total number of times each animal moved from one section of the exhibit to another (hereafter "transitions") during observations as a proportion of the total number of observational scans for each day. All proportional data were then arc-sine square root transformed. A MANOVA was used to test for the effects of individual (female and male okapi), time of day (AM or PM), and treatment (presence or absence of food patches) on the proportion of time spent on the two behavioral categories and on transitions.

Landscape of comfort. To test for effects of patch location on GUDs for the okapi, GUD data were analyzed using a general linear model (SYSTAT 13, San Jose, CA, U.S.A.). Date was nested within individual for the analysis, with GUD as the dependent variable, and patch location and individual (male or female okapi) as independent variables. We used days of each experiment as replicates rather than repeated measures because GUD measurements are independent from one day to the next (Troxell-Smith et al., 2016). A Fisher's Least Significant Difference (LSD) post-hoc test was used to identify differences between individual's use of patch locations, and to group patch locations into high (indicating an uncomfortable area), intermediate, and low (indicating a comfortable area) GUDs.

\section{Results}

\section{Time Budget/Behavioral Enrichment}

The main effect of treatment (presence or absence of food patches) significantly affected the okapi behavior, Wilk's $\lambda=0.78, F(3,68)=6.28, p=0.001$. Univariate $F$ tests found that food patches significantly increased both the proportion of time spent Foraging, $F(1,70)=9.16, p=0.003$, and the proportion of transitions within the exhibit, $F(1,70)=6.49, p=0.013$, for the two okapis. Food patches did not significantly influence the proportion of time spent on Repetitive behaviors, $F(1,70)=0.56, p=$ 0.458 ; Figure 2.

There was also a significant main effect of individual (male or female) on behavior, Wilk's $\lambda=$ $0.85, F(3,68)=3.87, p=0.013$. Univariate tests revealed that the female spent a significantly larger proportion of time on Repetitive behaviors than the male, $F(1,70)=9.80, p=0.003$. The two okapis did not differ in their proportion of time spent Foraging, $F(1,70)=1.71, p=0.196$, or their exhibit transitions, $F(1,70)=1.29, p=0.261$.

Time of day (AM vs. PM) also significantly influenced the okapis' behaviors, Wilk's $\lambda=0.86$, $F(3,68)=3.85, p=0.013$. Univariate tests revealed that time of day influenced Foraging behavior, $F(1$, $70)=10.62, p=0.002$, with more Foraging occurring in the morning vs. the evening. Time of day did not influence proportion of time spent Repetitive, $F(1,70)=0.04, p=0.846$, or the proportion of exhibit transitions, $F(1,70)=0.27, p=0.604$. There was no significant interaction effect between individual okapi and the presence or absence of food patches, Wilk's $\lambda=0.95, F(3,68)=1.22, p=0.310$, time of day and treatment, Wilk's $\lambda=0.97, F(3,68)=0.75, p=0.524$, or between individual and time of day, Wilk's $\lambda=0.90, F(3,68)=2.59, p=0.060$. The three-way interaction between individual, time of day, and treatment was also not significant, Wilk's $\lambda=0.94, F(3,68)=1.48, p=0.228$. 


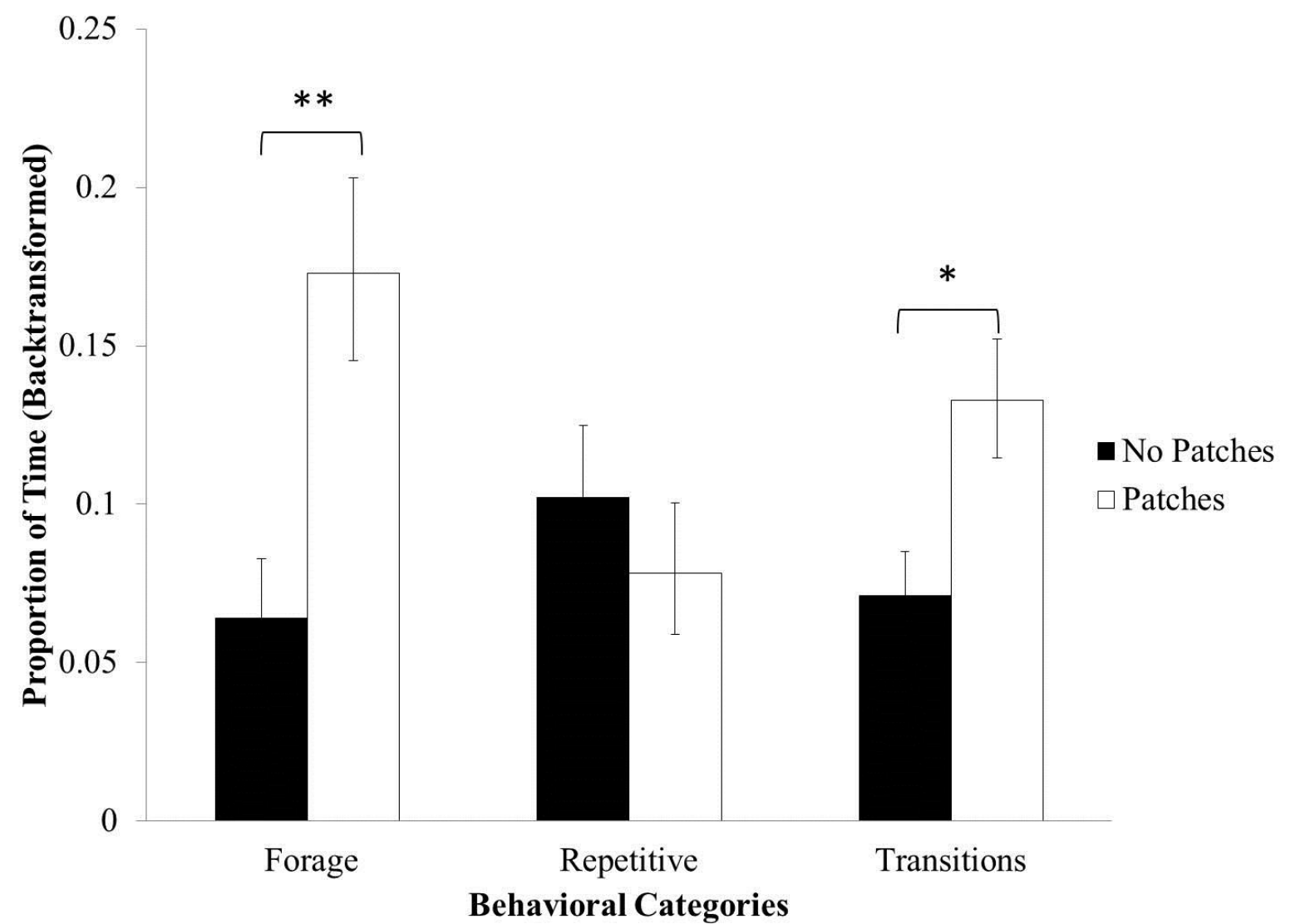

Figure 2. Pooled mean back-transformed proportion of time ( \pm SEM) spent in the Foraging and Repetitive behavioral categories, and proportion of Exhibit Transitions in each treatment condition (with and without food patches). Patch treatment significantly increased proportion of time spent Foraging and proportion of exhibit transitions, but had no significant effect on proportion of time spent in Repetitive behaviors. Asterisks indicate level of significance (*p<0.025; ** $p<0.01)$.

\section{Landscape of Comfort}

Food patch location significantly influenced GUDs, $F(23,345)=5.95 ; p<0.001$. Although the overall GUDs did not differ significantly between the male and female okapis, $F(1,345)=0.28 ; p=$ 0.074 , there was a significant interaction between individual and patch location on GUDs, $F(23,345)=$ $4.89 ; p<0.001$ (Figures 3A and 4A), suggesting that the male and female differed in their landscapes of comfort. A Fisher's LSD revealed that the male and female okapi significantly varied in their use of nine out of 24 food patches (patches 1, 3, 5, 9, 10, 11, 17, $18 \& 20$ ). Overall, the female okapi had high GUDs (indicating area of discomfort; mean GUD > $80 \mathrm{~g}$ remaining) in six of 24 food patches, intermediate GUDs (between $50-79 \mathrm{~g}$ ) in 13 patches, and low GUDs (indicating an area of comfort; < $49 \mathrm{~g}$ ) in five patches (Figure 3B). The male okapi had high GUDs (> $80 \mathrm{~g}$ remaining) in seven of 24 food patches, intermediate GUDs (between $50-79 \mathrm{~g}$ ) in 14 patches, and low GUDs $(<49 \mathrm{~g})$ in four patches (Figure 4B). GUDs were similarly high (indicating discomfort) for both okapis in three locations (Patches 2, 7, and 22), whereas both okapis had low GUDs (indicating comfort) in three other locations (Patches 1, 12, and 15). For only one location (Patch 5) the female GUD suggested relative comfort, but the male's GUD suggested relative discomfort. High GUDs indicate the okapis were less comfortable in approximately $25 \%$ of their exhibit space. 


\section{A)}

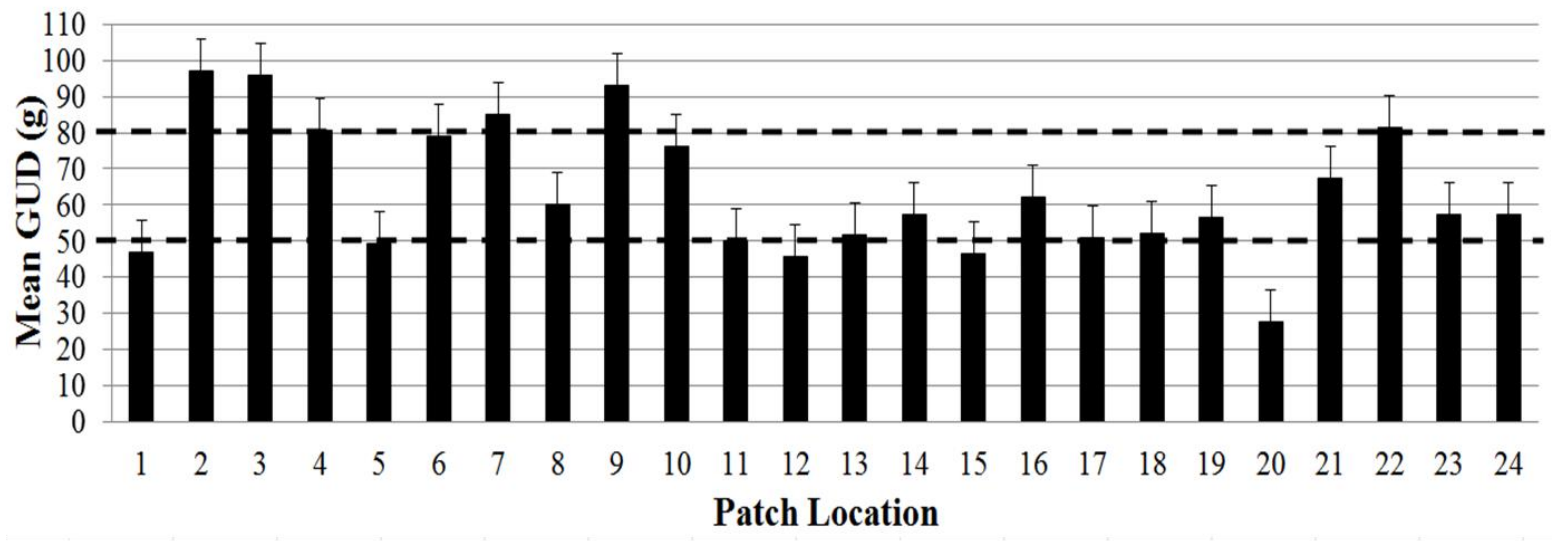

B)

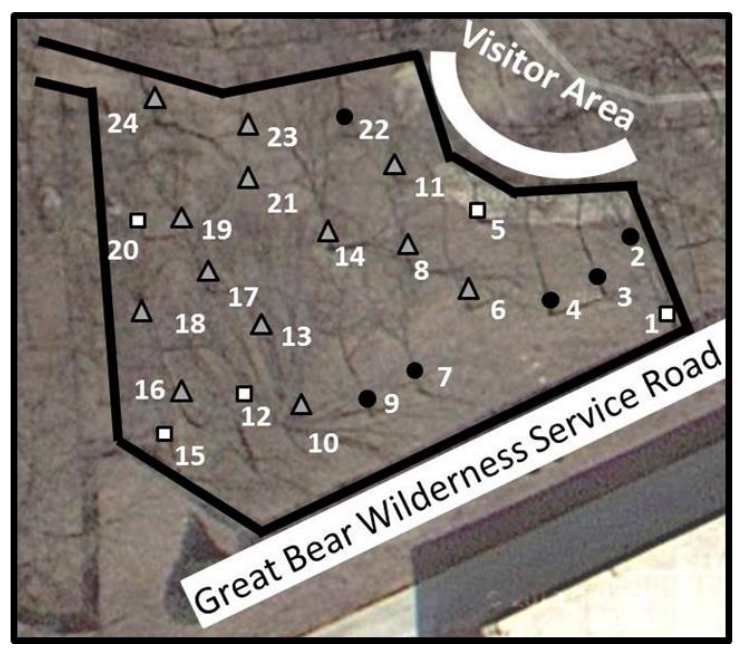

Figure 3. A) Pooled mean GUD ( \pm SEM) for the female okapi. Patch location had a significant effect on GUDs $(p<0.001)$. Dashed lines indicate cut-offs for high (avg GUD > $80 \mathrm{~g}$ ), intermediate (avg GUD between 79-50 g), and low GUDs (avg GUD < $49 \mathrm{~g}$ ). GUDs in patches $2,3,4,7,9$, and 22 were significantly higher than other locations, and GUDs in patches 1, 5, 12, 15, and 20 were significantly lower than other locations. B) Corresponding Google Earth map of the female okapi's "landscape of comfort" within the exhibit at the Brookfield Zoo. Each shape represents a foraging station, and shapes are color-coded to represent pooled mean GUDs at patch location: black circles = high GUD (avg GUD > 80 g); gray triangles = intermediate GUD (avg GUD between $79-50 \mathrm{~g}$ ); white squares = low GUD (avg GUD $<49$ g). 
A)

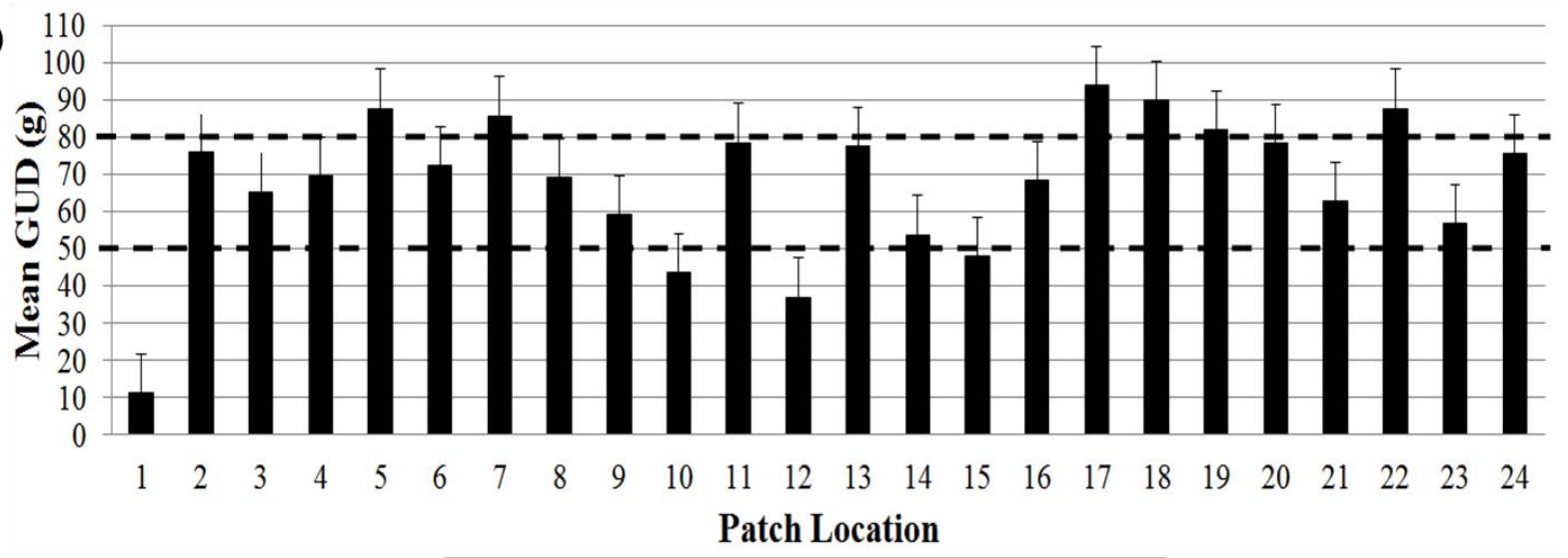

B)

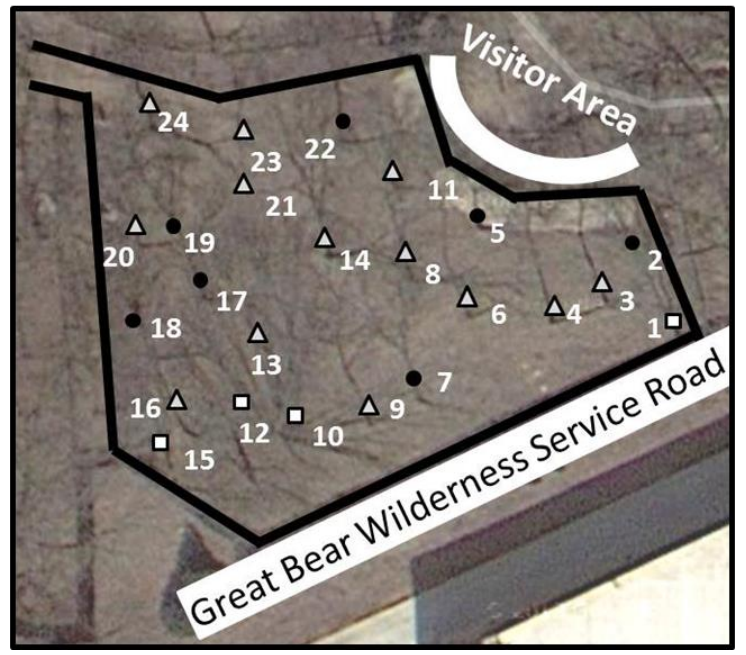

Figure 4. A) Pooled mean GUD ( \pm SEM) for the male okapi. Patch location had a significant effect on GUDs $(p<0.001)$. Dashed lines indicate cut-offs for high (avg GUD > $80 \mathrm{~g}$ ), intermediate (avg GUD between 79-50 g), and low GUDs (avg GUD < $49 \mathrm{~g}$ ). GUDs in patches 2, 5, 7, 17, 18, 19, and 22 were significantly higher than other locations, and GUDs in patches 1, 10, 12, and 15 were significantly lower than other locations. B) Corresponding Google Earth map of the male okapi's "landscape of comfort" within the exhibit at the Brookfield Zoo. Each shape represents a foraging station, and shapes are color-coded to represent pooled mean GUDs at patch location: black circles = high GUD (avg GUD > $80 \mathrm{~g}$ ); gray triangles = intermediate GUD (avg GUD between $79-50 \mathrm{~g}$ ); white squares = low GUD (avg GUD < 49 g).

\section{Discussion}

Using a combination of behavioral observations, food patches, and foraging ecology, we evaluated and compared the behavior and spatial preferences of two adult okapi within the same exhibit. Although our study was based on the responses of two individuals that vary in sex, age, and lifeexperience, we nevertheless found several interesting results. Behavioral results indicate that providing food patches significantly increased the proportion of time both okapi spent foraging, and increased within-exhibit movement, but there was no significant effect of food patches on repetitive behavior. Furthermore, while there were some similarities between landscapes of comfort between the two individuals, there were also some striking individual differences in spatial preferences within the same exhibit space.

As predicted, food patches provided valuable insight regarding how the individual okapi perceived the same exhibit space. Namely, the food patches highlighted several areas of discomfort that 
could have important impacts on animal welfare. For example, the female okapi had high GUDs in six of the 24 food patches, suggesting that $25 \%$ of the exhibit was perceived as risky or uncomfortable from the female's perspective. Interestingly, the majority of the food patches with high GUDs were located directly adjacent to a service road bordering the exhibit. Given that wild okapi are native to remote, dense rainforest areas in the Democratic Republic of Congo (Hart, 2013; Hart \& Hart, 1988), it is possible that the female okapi was highly sensitive to the general human activity and/or vehicle noise occurring along the service road. These hypotheses could be tested further by measuring sound decibel output near vs. away from the service road, reducing human and vehicle traffic, or by experimentally increasing visual barriers (Troxell-Smith \& Miller, 2016) along the service road fence-line to create a more enclosed, quiet space. The exhibit preferences and aversions of the male okapi on the other hand, were largely different from those of the female. Like the female, the male okapi also left high GUDs in seven out of 24 food patches, again suggesting that at least $25 \%$ of the okapi's exhibit was viewed as risky or uncomfortable from the male's perspective. Unlike the female, however, the majority of food patches with the highest GUDs were located either along the visitor area or toward the back of the exhibit. Previous studies suggest that visitor proximity may negatively impact zoo animal behavior and welfare (see Davey, 2007 for a review; Quadros, Goulart, Passos, Vecci, \& Young, 2014; Suárez, Recuerda, \& Arias-de-Reyna, 2017); it is therefore possible that the male okapi was more sensitive and possibly threatened by the proximity of human visitors compared to the female. This idea could be tested further by adding additional visual barriers or canopy cover along the exhibit barrier between the visitors and the okapi.

In addition to revealing the environmental preferences of each individual, food patches provided valuable behavioral benefits as well. As hypothesized, both okapi significantly increased the proportion of time foraging, and the proportion of transitions within the exhibit when provided with food patches compared to standard feeding conditions. These results suggest that food patches provide important behavioral benefits to the okapi. In the wild, okapi spend the majority of daylight hours moving within their home ranges to acquire their food (Hart \& Hart, 1988; Lindsey, Green, \& Bennett, 1999). Regimented feeding schedules in captivity, however, drastically reduce the control captive animals have over their foraging behaviors, such as where, when, and how thoroughly to forage (Morgan \& Tromborg, 2007; Newberry, 1995). In fact, a recent study suggests that whereas most captive okapi are provided with browse throughout their day, they are fed the bulk of their main, pelletized diet only once or twice a day (Bennett, Torgerson-White, Fripp, Watters, \& Petric, 2015), which is directly at odds with their natural foraging ecology and behavior. Thus, provisioning okapi, and even other animals, with food patches may also provide individuals with more control over their foraging efforts throughout the day. Animals can choose when and where to eat, leave and return to multiple food patches throughout the day, and even avoid patches in uncomfortable areas, allowing for a foraging experience more similar to that in the wild. The inedible substrate (wiffle balls) in the food patches increases the time it takes for animals to forage compared to providing food in a simple trough or container. Moreover, by establishing several foraging locations throughout the exhibit, animals are encouraged to travel more to acquire their food as opposed to feeding from a single spot.

Although there appeared to be a slight decrease in repetitive behaviors when food patches were present, the effect was not statistically significant. When provided with food patches, the proportion of time the female okapi spent performing repetitive behaviors decreased slightly from approximately $39.5 \%$ during traditional foraging methods to $32.3 \%$ with food patches. The male okapi also slightly decreased time spent in repetitive behaviors (from $14.8 \%$ to 13.4\%) with food patches present. These results, combined with previous research suggesting that increasing feeding frequency decreased the performance of repetitive licking behavior in okapi (Bashaw, Tarou, Maki, \& Maple, 2001), lead us to suggest that further investigation into the potential impact that food patches might have on repetitive behavior may be warranted. Increasing both the number of animals observed and the number of observation days in each condition may further elucidate any potential effects.

Because our study focused on two okapi with varied backgrounds, it is difficult to generalize their results to the broader okapi population. However, preferences at an individual level are still important to evaluate and acknowledge, particularly for animals in captivity. Individuals within a species are known to 
vary in how they perceive and respond to their captive exhibit space, and therefore the degree of wellbeing they experience (Tetley \& O'Hara, 2012). Thus, techniques that allow caretakers to evaluate individual differences in exhibit perceptions are extremely important to aid in improving welfare outcomes. In the current study, use of food patches not only allowed our two okapi to reveal their individual preferences and aversions in response to the same exhibit space, but also served as a valuable behavioral enrichment tool. However, additional avenues of research remain. An important next step will be to use the established landscapes of comfort to directly inform changes to exhibit design. In the present study, adding canopy cover and visual barriers to the existing okapi exhibit would be fairly inexpensive modifications to quickly address the specific aversions demonstrated by the male and female okapi, thus tailoring the shared exhibit space to each individual's needs. Such changes could also allow for more informed large-scale and permanent modifications in the future. Furthermore, additional evaluation of the possible impact that food patches may have on behavioral measures can help to further establish the potential of food patches to inform welfare-related decisions for animals in captivity.

\section{Conclusions}

Overall, we suggest that implementation of foraging ecology and food patches within captive environments can help to achieve important goals of effective behavioral enrichment (including increasing targeted foraging behaviors and exhibit utilization, and the potential to reduce repetitive behaviors) while simultaneously providing key insight into individual differences in animal preference, state, and welfare. Food patches are an economic and versatile enrichment option that not only improve animal behavioral outcomes, but also provide valuable information about animal exhibit perceptions directly from their perspective. Such information can then be applied to inform a variety of welfarerelated decisions not only for okapi, but for other zoo species as well.

\section{Acknowledgements}

The authors thank Joan Daniels, Amy Roberts, and the entire Brookfield Zoo Habitat Africa Forest animal care team (particularly Diane Gierhahn, Tina Houchens, and Carla Thompson) for their valuable assistance in making this project possible. We also thank Lance Miller, Bill Zeigler and Alejandro Grajal for their continued support of our research, Kristie Charmoy for her assistance with data collection and entry, and Gaclyn Antar for her valuable assistance in food patch construction. Financial support for this study was provided by a UIC Ecology and Evolution Elmer Hadley Graduate Research Grant, and a NSF Graduate Research Fellowship (grant \#DGE 097994), awarded to SMTS.

\section{References}

Altmann, J. (1974). Observational study of behavior: Sampling methods. Behaviour, 49, 227-266.

Bashaw, M. J., Tarou, L. R., Maki, T. S., \& Maple, T. L. (2001). A survey assessment of variables related to stereotypy in captive giraffe and okapi. Applied Animal Behaviour Science, 73, 235-247.

Bennett, C., Torgerson-White, L., Fripp, D., Watters, J., \& Petric, A. (2015). A multi-institutional assessment of factors influencing locomotion and pacing in captive Okapis (Okapia johnstoni). Journal of Applied Animal Welfare Science, 18, S43-S61.

Bodmer, R. E., \& Rabb, G. B. (1992). Okapia johnstoni. Mammalian Species, 422, 1-8.

Bond, J., \& Lindburg, D. (1990). Carcass feeding of captive cheetahs (Acinonyx jubatus): The effects of a naturalistic feeding program on oral health and psychological well-being. Applied Animal Behaviour Science, 26, 373-382.

Brown, J. (1988). Patch use as an indicator of habitat preference, predation risk, and competition. Behavioral Ecology and Sociobiology, 22, 37-47.

Carlstead, K. (1996). Effects of captivity on the behavior of wild mammals. In D. G. Kleiman, M. E. Allen, K. V Thompson, S. Lumpkin, \& H. Harris (Eds.), Wild mammals in captivity: Principles and techniques (1st Ed., pp. 317-333). Chicago, IL: University of Chicago Press. 
Carlstead, K., Seidensticker, J., \& Baldwin, R. (1991). Environmental enrichment for zoo bears. Zoo Biology, 16, 316.

Chamove, A. (1989). Environmental enrichment: A review. Animal Technology, 40, 155-178.

Charnov, E. L. (1976). Optimal foraging, the marginal value theorem. Theoretical Population Biology, 9, 129-136.

Davey, G. (2007). Visitors' effects on the welfare of animals in the zoo: A review. Journal of Applied Animal Welfare Science, 10, 169-183.

Hare, V. J., Ripsky, D., Battershill, R., Bacon, K., Hawk, K., \& Swaisgood, R. R. (2003). Giant panda enrichment: Meeting everyone's needs. Zoo Biology, 22, 401-416.

Hart, J. (2013). Okapia johnstoni. In J. Kingdon \& M. Hoffmann (Eds.), Mammals of Africa Volume 6: Pigs, deer, giraffe, bovids and hippos (pp. 110-115). London, UK: Bloomsbury Publishing.

Hart, J., \& Hart, T. (1988). A summary report on the behaviour, ecology and conservation of the okapi (Okapia johnstoni) in Zaire. Acta Zoologica et Pathologica Antverpiensia, 80, 19-28.

Herbers, J. (1981). Time resources and laziness in animals. Oecologia, 49, 252-262.

Howell-Stephens, J. A. (2012). Assessing welfare of armadillos using hormonal \& foraging indicators, and patch use in Argentinean birds (Doctoral dissertation). Retrieved from ProQuest Dissertations Publishing. (Order No. 3551885).

Ings, R., Waran, N., \& Young, R. (1997). Effect of wood-pile feeders on the behaviour of captive bush dogs (Speothos venaticus). Animal Welfare, 6, 145-152.

Kistler, C., Hegglin, D., Würbel, H., \& König, B. (2009). Feeding enrichment in an opportunistic carnivore: The red fox. Applied Animal Behaviour Science, 116, 260-265.

Lindsey, S. L., Green, M. N., \& Bennett, C. L. (1999). The Okapi: Mysterious animal of Congo-Zaire (1st Ed.). Austin, TX: University of Texas Press.

Mason, G. (1991). Stereotypies: A critical review. Animal Behaviour, 41, 1015-1037.

Mason, G., \& Latham, N. (2004). Can't stop, won't stop: Is stereotypy a reliable animal welfare indicator? Animal Welfare, 13, S57-69.

Mason, G. J., \& Veasey, J. S. (2010). How should the psychological well-being of zoo elephants be objectively investigated? Zoo Biology, 29, 237-255.

McPhee, M. E. (2002). Intact carcasses as enrichment for large felids: Effects on on- and off-exhibit behaviors. Zoo Biology, 21, 37-47.

McPhee, M. E. (2003). Generations in captivity increases behavioral variance: Considerations for captive breeding and reintroduction programs. Biological Conservation, 115, 71-77.

McPhee, M. E., \& Carlstead, K. (2010). The importance of maintaining natural behaviors in captive mammals. In D. G. Kleiman, K. V. Thompson, \& C. Kirk Baer (Eds.), Wild mammals in captivity: Principles and techniques for zoo management (2nd Ed., pp. 303-313). Chicago, IL: University of Chicago Press.

Mogerman, J. E. E. H. (2011). Zoo foraging ecology: Patch use and giving-up densities as tools for animal care (Doctoral dissertation). Retrieved from ProQuest Dissertations Publishing. (Order No. 3417306).

Morgan, K. N., \& Tromborg, C. T. (2007). Sources of stress in captivity. Applied Animal Behaviour Science, 102, 262-302.

Newberry, R. C. (1995). Environmental enrichment: Increasing the biological relevance of captive environments. Applied Animal Behaviour Science, 44, 229-243.

Quadros, S., Goulart, V. D. L., Passos, L., Vecci, M. A. M., \& Young, R. J. (2014). Zoo visitor effect on mammal behaviour: Does noise matter? Applied Animal Behaviour Science, 156, 78-84.

Réale, D., Reader, S. M., Sol, D., McDougall, P. T., \& Dingemanse, N. J. (2007). Integrating animal temperament within ecology and evolution. Biological Reviews of the Cambridge Philosophical Society, 82, 291-318.

Ruskell, A. D., Meiers, S. T., Jenkins, S. E., \& Santymire, R. M. (2015). Effect of Bungee-carcass enrichment on behavior and fecal glucocorticoid metabolites in two species of zoo-housed Felids. Zoo Biology, 34, 170-177.

Ryan, E. B., Proudfoot, K. L., \& Fraser, D. (2012). The effect of feeding enrichment methods on the behavior of captive Western lowland gorillas. Zoo Biology, 31, 235-241.

Schneider, M., Nogge, G., \& Kolter, L. (2014). Implementing unpredictability in feeding enrichment for Malayan sun bears (Helarctos malayanus). Zoo Biology, 9, 1-9.

Shepherdson, D. (2010). Principles of and research on environmental enrichment for mammals. In D. G. Kleiman, K. V. Thompson, \& C. Kirk Baer (Eds.), Wild mammals in captivity: Principles and techniques for zoo management (2nd Ed., pp. 62-67). Chicago, IL: University of Chicago Press.

Shyne, A. (2006). Meta-analytic review of the effects of enrichment on stereotypic behavior in zoo mammals. Zoo Biology, 25, 317-337.

Suárez, P., Recuerda, P., \& Arias-de-Reyna, L. (2017). Behaviour and welfare: The visitor effect in captive felids. 
Animal Welfare, 26, 25-34.

Swaisgood, R., \& Shepherdson, D. (2005). Scientific approaches to enrichment and stereotypies in zoo animals: What's been done and where should we go next? Zoo Biology, 24, 499-518.

Tetley, C., \& O'Hara, S. (2012). Ratings of animal personality as a tool for improving the breeding, management, and welfare of zoo animals. Animal Welfare, 21, 463-476.

Troxell-Smith, S. M., \& Miller, L. J. (2016). Using natural history information for zoo animal management: A case study with okapi (Okapia johnstoni). Journal of Zoo and Aquarium Research, 4, 38-41.

Troxell-Smith, S. M., Tutka, M. J., Albergo, J. M., Balu, D., Brown, J. S., \& Leonard, J. P. (2016). Foraging decisions in wild versus domestic Mus musculus: What does life in the lab select for? Behavioural Processes, $122,43-50$.

Troxell-Smith, S. M., Whelan, C. J., Magle, S. B., \& Brown, J. S. (in press). Zoo foraging ecology - development and assessment of a welfare tool for captive animals. Animal Welfare.

Watters, J. V., Miller, J. T., \& Sullivan, T. J. (2011). Note on optimizing environmental enrichment: A study of fennec fox and zoo guests. Zoo Biology, 30, 647-654. 